\title{
Spinons in a Crossed-Chains Model of a 2D Spin Liquid
}

\author{
Oleg A. Starykh ${ }^{1}$, Rajiv R. P. Singh ${ }^{2}$, and Gregory C. Levine ${ }^{1}$ \\ ${ }^{1}$ Department of Physics and Astronomy, Hofstra University, Hempstead, NY 11549 \\ ${ }^{2}$ Department of Physics, University of California, Davis, CA 95616
}

(February 11, 2002; cond-mat/0106260)

\begin{abstract}
Using Random Phase Approximation, we show that a crossed-chains model of a spin-1/2 Heisenberg spins, with frustrated interchain couplings, has a non-dimerized spin-liquid ground state in 2D, with deconfined spinons as the elementary excitations. The results are confirmed by a bosonization study, which shows that the system is an example of a 'sliding Luttinger liquid'. In an external field, the system develops an incommensurate field-induced long range order with a finite transition temperature.
\end{abstract}

PACS: 75.10.Jm, 75.30.Kz, 75.40.Gb

Geometrically frustrated magnets have attracted strong interest in recent years, primarily because they provide us with a potentially direct route to the actively sought "spin liquid" phases of two- (2d) and threedimensional (3d) strongly correlated electron systems. Begun in the 50's [1], this line of research was dramatically revitalized by Anderson's proposal [2] that such a liquid of spin singlets is, upon hole doping, adiabatically connected to the ground state of layered cuprate superconductors. Whether or not this is so remains to be seen, but the following "question of principle" — can the ground state of a $2 \mathrm{~d}$ or $3 \mathrm{~d}$ magnetic system be liquid like? - has become one of the most debated questions in condensed matter physics. Significant insight into this problem has been gained recently via the dual description of frustrated antiferromagnets in terms of gauge Ising models [3 6]. In this paper we, however, take a more direct, experimentally-motivated approach, following recent work by Bocquet et al. [7] and an earlier paper by two of us and P. J. Freitas [8].

The "spin liquid" (also known as "resonating valence bond", or RVB, phase) is defined as a liquid of singlet spin pairs covering the lattice, and is characterized by the absence of long-range order (LRO), unbroken spin rotational and translational symmetries, and elementary excitations with fractional spin $1 / 2$ (spinons). Its physical relevance has been highlighted by the experimental observation of spin liquid-like behavior in the kagomé lattice compound $\mathrm{SrCr}_{8-x} \mathrm{Ga}_{4+x} \mathrm{O}_{19}$ [9] and several pyrochlore antiferromagnets [10], e.g. $\mathrm{CsNiCrF}_{6}$. Both materials are based on frustrated units - triangles (kagomé) and tetrahedra (pyrochlore) - combined in site sharing arrangements, which leave the spins in the classical ground-states highly underconstrained [11].

On the other hand, it is well known that a spin liquid state is realized in the antiferromagnetic Heisenberg chain (HAFC). It is then natural to try to build up $2 \mathrm{~d}$ (or 3d) spin liquid state from HAFCs - the theoretical investigation of this possibility is the subject of this paper.

Elementary excitations of the single chain are deconfined gapless spin- $1 / 2$ spinons, which can be visualized as domain walls separating domains of different orientation (up and down) of staggered magnetization $\vec{n}(x)=$ $(-1)^{x} \vec{S}(x)$ along the chain. Consider now a parallel array of HAFCs coupled by unfrustrated exchange $J_{\perp}$ in the direction transverse to the chain: no matter how small $J_{\perp}$ is, it immediately leads to the confinement of spinons because the energy of two domain walls grows linearly with separation between them, Fig.1a. This is an intuitive reason, confirmed by detailed calculations [12], for the stabilization of LRO and appearance of spin-1 magnons (which are bound states of two spinons) in this situation. It is clear, however, that this argument fails if each spin is coupled, in a transverse direction, to the zerospin combination of spins on neighboring chains, i.e. if the transverse coupling is frustrating. Motivated by this simple argument and recent experiments on $\mathrm{Cs}_{2} \mathrm{CuCl}_{4}$ 13. and, especially, $\mathrm{Na}_{2} \mathrm{Ti}_{2} \mathrm{Sb}_{2} \mathrm{O}$ [14], we investigate the crossed-chains model [8] (CCM) - the two-dimensional Heisenberg model on the lattice shown in Fig.1b,

$$
H_{c c}=J \sum_{\langle i, j\rangle} \vec{S}_{i} \vec{S}_{j}+J^{\prime} \sum_{\langle i, j\rangle} \vec{S}_{i} \vec{S}_{j}
$$

The intra-chain exchange $J$ couples neighboring spins
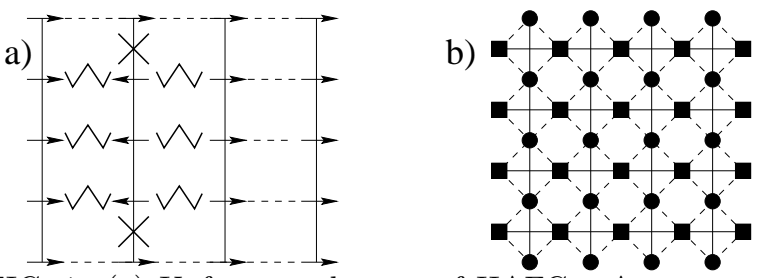

FIG. 1. (a) Unfrustrated array of HAFCs. Arrows represent staggered magnetization $\vec{n}(x)$. Solid (dashed) lines stand for exchange $J\left(J_{\perp}\right)$, whereas strings mark broken $J_{\perp}$ bonds. Domain walls are indicated by crosses. (b) The crossed-chains lattice. Filled circles (squares) indicate spins on the vertical (horizontal) chains. Solid (dashed) lines denote intra-chain $J$ (inter-chain $J^{\prime}$ ) exchange interactions.

in a given row (column) which form a square mesh of HAFC along the $\mathrm{X}$ and $\mathrm{Y}$ axes, whereas the inter-chain $J^{\prime}$ is the nearest neighbor coupling between spins in rows 
and columns. The crossed-chain Hamiltonian interpolates between decoupled HAFCs $\left(J^{\prime}=0\right)$, the $2 \mathrm{~d}$ pyrochlore lattice $\left(J^{\prime}=J\right)$, and $45^{\circ}$-rotated square lattice $(J=0)$ 15. Notice that, when neighboring spins are antiparallel along the chains, the inter-chain contribution to the effective field acting on a given spin is zero.

1. RPA analysis of the crossed-chains model in the weak-coupling limit $J^{\prime} \ll J$. The defining feature of the HAFC is the absence of coherent spin-1 magnon excitations, already mentioned above. The spectrum of magnetic excitations consists of spin-1/2 noninteracting spinons, and the usual spin-1 magnon is an incoherent two-spinon excitation. It is then natural to use an approach which properly accounts for this important onedimensional feature in the "decoupled" limits $J^{\prime} / J \rightarrow 0$ and/or $T \gg J^{\prime}$. This is provided by a Random-PhaseApproximation (RPA) 16, 17, 8,7] which has the meaning of an expansion in the inverse coordination number of the lattice $z_{\perp}(=4$ for CCM). Applied to the crossedchains lattice of Fig.1b, it gives the following result for the dynamical spin susceptibility of CCM

$$
\begin{aligned}
& \chi_{R P A}\left(\omega, k_{x}, k_{y}\right)=\left(\chi_{1}\left(\omega, k_{x}\right)+\chi_{1}\left(\omega, k_{y}\right)-2 J^{\prime}(\vec{k}) \times\right. \\
& \left.\chi_{1}\left(\omega, k_{x}\right) \chi_{1}\left(\omega, k_{y}\right)\right)\left(1-\left(J^{\prime}(\vec{k})\right)^{2} \chi_{1}\left(\omega, k_{x}\right) \chi_{1}\left(\omega, k_{y}\right)\right)^{-1}
\end{aligned}
$$

where momentum $\vec{k}=\left(k_{x}, k_{y}\right)$ is measured from the antiferromagnetic momentum $(\pi, \pi)$ (lattice spacing is set to unity). The dynamical susceptibility of a single horizontal $\left(k_{x}\right)$ and vertical $\left(k_{y}\right)$ chain, $\chi_{1}(\omega, k)$, is known exactly (for details see, e.g., [7]),

$$
\begin{aligned}
& \chi_{1}(\omega, k)=-\frac{\sqrt{\ln (\Lambda / T)}}{2(2 \pi)^{3 / 2} T} \rho_{\eta}\left(\frac{\omega-v k}{4 \pi T}\right) \rho_{\eta}\left(\frac{\omega+v k}{4 \pi T}\right), \\
& \rho_{\eta}(x)=\frac{\Gamma\left(\frac{\eta}{4}-i x\right)}{\Gamma\left(1-\frac{\eta}{4}-i x\right)}
\end{aligned}
$$

where $\Gamma(x)$ is the Gamma function, $\Lambda=24.27 J$ [18 is the high-energy cutoff, numerical value of the pre-factor was calculated in 19, $\eta=1$ for the isotropic HAFC, and $v=\pi J / 2$ is the spinon velocity.

The specific lattice structure of CCM is encoded in the Fourier transform of the inter-chain exchange interaction

$$
J^{\prime}(\vec{k})=2 J^{\prime} \sin \left(k_{x} / 2\right) \sin \left(k_{y} / 2\right),
$$

and its frustrating character is clear from the fact that $J^{\prime}(0,0)=0$.

An ordering instability, if any, should show up as a divergence in $\chi_{R P A}\left(0, \vec{k}_{0}\right)$ at some critical temperature $T_{0}$ and momentum $\vec{k}_{0}=\left(k_{0}, \pm k_{0}\right)$ along two diagonal directions where antiferromagnetic fluctuations are the strongest. Extremizing the denominator of (2) with respect to $k_{0}$ one arrives at the following implicit equation for the ratio $x_{0}=k_{0} /\left(4 \pi T_{0}\right)(\Psi(x)$ is the digamma function)

$$
\frac{1}{x_{0}}+\pi \tanh \left(2 \pi x_{0}\right)-2 \operatorname{Im} \Psi\left(\frac{1}{4}+i x_{0}\right)=0 .
$$

This equation is very similar to the one obtained for the spin model describing $\mathrm{Cs}_{2} \mathrm{CuCl}_{4}[7]$ - in that case the coefficient of the first term in (5) is 2 times smaller. This minor difference is, however, extremely important: unlike the $\mathrm{Cs}_{2} \mathrm{CuCl}_{4}$ model, Eq.(5) has no solution. Thus, RPA analysis predicts no ordering instability down to, and including, $T=0$ : the crossed chains remain decoupled and provide us with an example of two-dimensional spin liquid with deconfined spinons as elementary excitations. The ineffectiveness of $J^{\prime}$ in destabilizing $1 \mathrm{~d}$ behavior of the crossed-chains is clearly seen from Fig.2 where we compare $\chi_{R P A}(\omega, k, k)(2)$ with twice the susceptibility of a single chain $\chi_{1}(\omega, k)(3)$. Even for unrealistically large $J^{\prime}=J$ the difference between these two susceptibilities is hardly observable. The same is true for corresponding structure factors $S_{\nu}(\omega, k)=-\left(1-e^{-\omega / T}\right)^{-1} \operatorname{Im} \chi_{\nu}(\omega, k, k)$ $(\nu=\mathrm{RPA}, 1)$ which are compared in Fig.3.

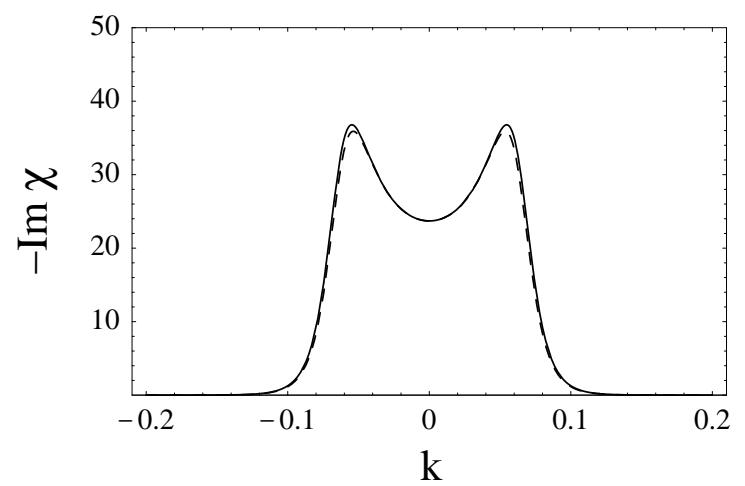

FIG. 2. $-\operatorname{Im} \chi_{R P A}(\omega, k, k)$ (solid line) and $-2 \times \operatorname{Im} \chi_{1}(\omega, k)$ (dashed line) vs. $k$ for $J^{\prime}=J, \omega=0.1 J, T=0.01 J$.

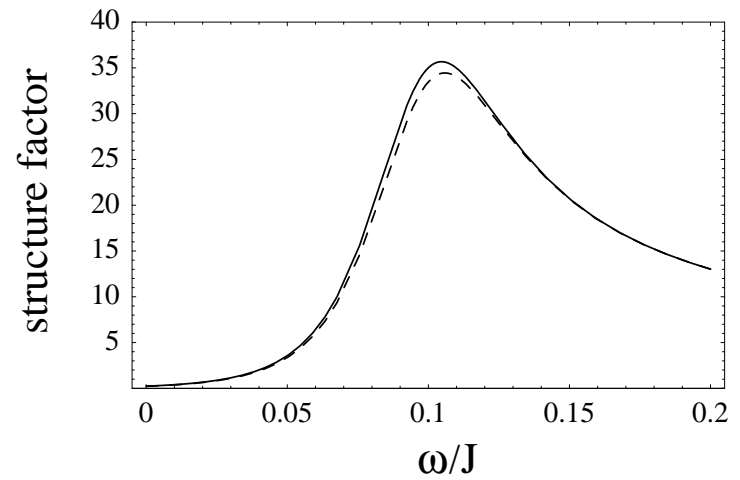

FIG. 3. $S_{R P A}(\omega, k)$ (solid line) and $2 \times S_{1}(\omega, k)$ (dashed line) vs. $\omega / J . k=0.06$ and other parameters as in Fig. 2 .

The reason for this is purely geometrical and is best illustrated at $T=0$ : the $1 / k$ divergence of $\chi_{1}(\omega=0, k)$, Eq.(3), is suppressed by the $k^{2}$ factor from $J^{\prime}(\vec{k})$, Eq.(41). This, again, should be contrasted with the $\mathrm{Cs}_{2} \mathrm{CuCl}_{4}$ lattice where the inter-chain exchange scales as $k$ and fails to suppress a remaining weakly diverging factor 
$\sqrt{\ln (1 /|k|)}$ due to a marginally irrelevant Umklapp interaction, which eventually does lead to the instability at some incommensurate wavevector [7].

Going back to the crossed-chains model it should be noted that the ordering instability may, in principle, happen at some "noncritical" $\vec{k}_{0}$ in the bulk of the Brillouin zone (BZ) when the ratio $J^{\prime} / J$ exceeds some critical value. Analysis of this interesting possibility requires knowledge of $\chi_{1}(0, k)$ for all values of momentum inside the BZ, which is currently not available. However, there exist a closely related model for which the static susceptibility is known exactly for all momenta - the HaldaneShastry chain (HSC) 20]. At $T=0$ its susceptibility diverges as $1 / k$ near the antiferromagnetic momentum $\pi$, similar to the HAFC, and remains finite everywhere else, $\chi_{H S}(0, k)=\arccos |k| /\left(2 \pi v|k| \sqrt{1-k^{2}}\right)$. Replacing $\chi_{1}(0, k)$ in Eq. (22) with $\chi_{H S}(0, k)$ in order to get an idea of what may happen in the CCM as the ratio $J^{\prime} / J$ increases, we find an instability at $k_{0} \approx 0.647 \pi$ when the ratio of exchanges exceeds $\left(J^{\prime} / J\right)_{\text {crit }}=(\pi / 2)^{3} \approx 3.87$. This high value of $J_{\text {crit }}^{\prime}$ in the HS model on the crossedchains lattice gives additional support to the robustness of the spin liquid state.

2. Effect of magnetic field. Interesting behavior is expected to develop when the crossed-chains model is subjected to a uniform magnetic field $H$. An applied field breaks spin-rotational symmetry between transverse and longitudinal susceptibilities [7]. The transverse one remains dominated by excitations from the gapless states at $k=0$ whereas the maximum of the longitudinal susceptibility $\chi_{1}^{z z}$ shifts to the incommensurate points $k_{ \pm}= \pm \delta(H), \delta(H)=2 \pi M(H)$, where $M(H)$ is the magnetization of a single HAFC. Since $J^{\prime}(\vec{k})$ is not affected by the field, the divergence of $\chi_{1}^{z z}\left(0, k \rightarrow k_{ \pm}\right)$is not compensated by inter-chain exchange anymore and an ordering instability does develop. It signals a transition into an incommensurate field-induced ordered phase (FILRO). Both the ordering temperature $T_{0}$ and the ordering momentum $\vec{k}_{0}$ can be calculated from a properly generalized Eq.(2). Repeating steps that led to (5) we obtain ( $k_{0}$ is measured from $k_{ \pm}$now)

$$
\begin{aligned}
& \frac{2 \pi T_{0}}{v} \cot \left(\frac{k_{0} \pm \delta}{2}\right)+\frac{\pi \sinh \left(2 \pi x_{0}\right)}{\cosh \left(2 \pi x_{0}\right)-\cos \left(\frac{\pi}{2 \eta}\right)} \\
& -2 \operatorname{Im} \Psi\left(\frac{1}{4}+i x_{0}\right)=0 .
\end{aligned}
$$

This equation has to be solved simultaneously with an equation for $T_{0}$, which is simply the condition that denominator of $\chi_{R P A}^{z z}\left(0, \vec{k}_{0}\right)$ is equal to zero. Notice that both the scaling exponent $\eta(H)$ and the spinon velocity $v(H)$ decrease with magnetic field, in particular $\eta(H)=1 / 2$ for saturating field $H \geq 2 J$ [7]. However, analytical solution is still possible in the limit of weak applied field when $\delta(H) \rightarrow 0$ thanks to the following inequalities $v k_{0} \ll T_{0}, k_{0} \ll \delta$ which can be checked a posteriori. We find incommensurate ordering with $k_{0, \pm}= \pm c_{1} \delta^{(2 \eta+1) /(2 \eta-1)}$ developing at $T_{0}=c_{2} v\left(\delta \sqrt{J^{\prime} / v}\right)^{2 \eta /(2 \eta-1)}$, where $c_{1,2}$ are weakly $H$ dependent constants of order 1 . So that for $H \rightarrow 0$ the scaling is $k_{0, \pm} \sim \pm \delta^{3}, T_{0} \sim J^{\prime} \delta^{2}$. Notice that $2 \mathrm{~d}$ ordering occurs at momenta different from $k_{ \pm}$at which susceptibility has a maximum for independent chains, although the difference is probably too small to be observed experimentally. Observation of such a FILRO in materials such as $\mathrm{Na}_{2} \mathrm{Ti}_{2} \mathrm{Sb}_{2} \mathrm{O}$ would be a direct signature for the applicability of the coupled-chains model presented here.

3. Scaling analysis: sliding Luttinger liquid phase.

We now employ bosonization to investigate effect of the inter-chain interaction $V$ at finite temperature $T$. We consider the mesh made of equal number of horizontal $(h)$ and vertical $(v)$ chains, $N_{h}=N_{v}=N$, each of length $L=N a$, where $a$ is the lattice spacing. In the continuum limit each site spin is represented by a sum of uniform $\vec{J}$ and staggered $\vec{n}$ fields 21]. In addition, $J_{v / h}^{\nu}=J_{v / h, R}^{\nu}+$ $J_{v / h, L}^{\nu}$, where $J_{R / L}^{\nu}$ are chiral WZW currents. The interchain interaction reads

$$
V=g \int d x d y \vec{J}_{h}(x, y) \cdot \vec{J}_{v}(x, y)
$$

where $g=2 J^{\prime}$. Note that due to the geometry of the problem the staggered magnetization $\vec{n}$, which has scaling dimension $\Delta(\vec{n})=1 / 2$, does not show up in this equation. As a result, the scaling dimension of the integrand in $(7)$ is 2 . The bare Hamiltonian $H_{0}$ describes total of $2 N$ independent vertical and horizontal chains. From here follows the defining feature of our model: $J-J$ correlations are non-zero only for like currents (i.e. $h-h$ and $v-v$ ) of the same chirality (i.e. R-R and L-L) which belong to the same chain, e.g.

$$
\begin{aligned}
& \left\langle J_{v, R}^{\mu}(x, y, \tau) J_{v, R}^{\nu}(0,0,0)\right\rangle=a \delta(x) \delta_{\mu, \nu}(\pi T / v)^{2} \\
& \times \frac{1}{8 \pi^{2}}\left\{\frac{1}{\sin ^{2}[\pi T(v \tau+i y) / v]}+\frac{1}{\sin ^{2}[\pi T(v \tau-i y) / v]}\right\}
\end{aligned}
$$

and $\left\langle J_{h} J_{h}\right\rangle$ is obtained by replacing $v \rightarrow h, y \leftrightarrow x$. The 'same-chain' condition is contained in an important delta-function which appears with pre-factor $a$ because $\delta\left(x-x^{\prime}\right)=\delta_{n, n^{\prime}} / a$. Conformal invariance of $H_{0}$ was used to write (8) at finite $T$ [22,21].

Our idea is to consider corrections to the bare free energy of $2 N$ chains $F_{0} / L^{2}=\pi v /\left(2 a^{3}\right)-\pi T^{2} /(3 v a)$ (momentum cut-off $\Lambda=\pi / a$ was used to get the first term). First correction is $\delta F^{(2)}=-T\left\langle V^{2}\right\rangle / 2$ where

$$
\begin{aligned}
\left\langle V^{2}\right\rangle= & (g a)^{2} \int d x d y \int_{0}^{1 / T} d \tau d \tau^{\prime}\left\langle J_{h}(x, y, \tau) J_{h}\left(x, y, \tau^{\prime}\right)\right\rangle \\
& \times\left\langle J_{v}(x, y, \tau) J_{v}\left(x, y, \tau^{\prime}\right)\right\rangle
\end{aligned}
$$

Notice that due to delta-functions in (8) spacial coordinates of currents in (9) are forced to coincide. Shortdistance divergence of the integrand in (9) is regularized by $a$, leading to 


$$
\left\langle V^{2}\right\rangle=\left(\frac{g a L T}{4 v^{2}}\right)^{2} \int_{0}^{2 \pi} \frac{d \phi}{2 \pi[\cos \phi-\cosh (2 \pi T a / v)]^{2}}
$$

The resultant correction to the free energy is irrelevant,

$$
\delta F^{(2)} / L^{2}=-\frac{\pi v}{2 a^{3}}\left(\frac{g a}{8 \pi^{2} v}\right)^{2}\left\{1-\frac{1}{15}(2 \pi T a / v)^{4}\right\} .
$$

This can be understood as follows. In the weak-coupling limit, where each crossing can be treated independently from others, interaction $V$ acts at a point rather than along the entire length of two chains forming that particular crossing. This is the physical reason for the absence of integration over the relative spatial coordinates in (9). It is well known that for the point interaction marginal dimension separating relevant from irrelevant perturbations is 1 23. Since $\Delta(V)=2>1, V$ is irrelevant.

At this order the ground state of the CCM is that of decoupled spin chains. At $T=0$ there are no correlations between spins on different chains due to irrelevancy of $V$ but at finite $T$ weak inter-chain correlations with a square $C_{4 v}$ symmetry will be present [24], in qualitative agreement with our RPA expression (2). Dimensional estimate of higher order in $g$ corrections show that their contribution is at most marginal. This allows us to identify the CCM as a $S U(2)$ invariant spin liquid with deconfined spinons as elementary excitations. Following earlier works on models with $U(1)$ symmetry 25,24 it can be called a sliding Luttinger liquid.

We thank A. G. Abanov, L. Balents, F. Essler, A. Furusaki, M. P. A. Fisher, C. Kane, S. Sachdev and A. M. Tsvelik for helpful discussions and suggestions. This research is supported by ITP (Santa Barbara) Scholarship and an award from Research Corporation (O.A.S.) and by NSF grant DMR9986948 (R.R.P.S.).

Note added: Since the submission of our work numerical studies have further addressed the $J^{\prime}=J$ limit of the model (the $2 \mathrm{~d}$ pyrochlore lattice) [26]. They find a valence-bond crystal phase with a spin-gap. These results are not in contradiction with our findings. In fact, making the reasonable assumption that the ground state energy in the valence-bond crystal phase and the sliding Luttinger liquid phase is weakly dependent on $J^{\prime} / J$, leads to an estimate for a quantum phase transition between the two at $J^{\prime} / J=0.85$. This allows for a wide range $0<J^{\prime} / J<0.85$ of values where the sliding Luttinger liquid phase could exist.

[1] G. H. Wannier, Phys. Rev. 79, 357 (1950); R. M. F. Houtappel, Physica 16, 425 (1950).

[2] P. W. Anderson, Science 235, 1196 (1987).

[3] T. Senthil and M. P. A. Fisher, Phys. Rev. B 62, 7850 (2000).
[4] S. Sachdev and M. Vojta, J. Phys. Soc. Jpn. 69, Suppl. B, 1 (2000).

[5] R. Moessner, S. L. Sondhi, and E. Fradkin, condmat/0103396.

[6] N. Read and S. Sachdev, Phys. Rev. B 42, 4568 (1990).

[7] M. Bocquet et al., Phys. Rev. B 64, 094425 (2001).

[8] R. R. P. Singh, O. A. Starykh, and P. J. Freitas, J. Appl. Phys. 83, 7387 (1998).

[9] Y. J. Uemura et al., Phys. Rev. Lett. 73, 3306 (1994).

[10] M. J. Harris and M. P. Zinkin, Mod. Phys. Lett. B 10, 417 (1996).

[11] R. Moessner and J. T. Chalker, Phys. Rev. B 58, 12049 (1998).

[12] H. J. Schulz, Phys. Rev. Lett. 77, 2790 (1996).

[13] R. Coldea et al., Phys. Rev. Lett. 86, 1335 (2001).

[14] E. A. Axtell III et al., J. Solid State Chem. 134, 423 (1997).

[15] Within linear spin-wave approximation, the $T=0$ transition from the long-range-ordered state at $J / J^{\prime}=0$ to a quantum-disordered one takes place at $\left(J / J^{\prime}\right)_{\text {crit }} \approx 0.76$, see [8] and B. Canals, cond-mat/0102233.

[16] D. J. Scalapino, Y. Imry, and P. Pincus, Phys. Rev. B 11, 2042 (1975).

[17] D. Boies, C. Bourbonnais, and A. -M. S. Tremblay, Phys. Rev. Lett. 74, 968 (1995).

[18] V. Barzykin, Phys. Rev. B 63, 140412 (R) (2001).

[19] S. Lukyanov and A. B. Zamolodchikov, Nucl. Phys. B 493, 571 (1997).

[20] F. D. M. Haldane and M. R. Zirnbauer, Phys. Rev. Lett. 71, 4055 (1993).

[21] A. O. Gogolin, A. A. Nersesyan, A. M. Tsvelik, Bosonization and Strongly Correlated Systems, Cambridge University Press, Cambridge, 1998.

[22] S. Sachdev, Phys. Rev. B 50, 13006 (1994).

[23] C. L. Kane and M. P. A. Fisher, Phys. Rev. B 46, 15233 (1992).

[24] R. Mukhopadhyay, C. L. Kane, and T. C. Lubensky, Phys. Rev. B 64, 045120 (2001); Phys. Rev. B 63, 081103 (2001).

[25] I. E. Dzyaloshinskii and A. I. Larkin, Sov. Phys. JETP38, 202 (1974); H. J. Schulz, J. Phys. C 16, 6769 (1983); S. A. Kivelson, V. J. Emery, and E. Fradkin, Nature 393, 550 (1998); V. J. Emery et al., Phys. Rev. Lett. 85, 2160 (2000); A. Vishwanath and D. Carpentier, Phys. Rev. Lett. 86, 676 (2001).

[26] J.-B. Fouet et al., cond-mat/0108070. For earlier numerical studies see S. E. Palmer and J. T. Chalker, Phys. Rev. B 64, 094412 (2001). 